\title{
Modeling the process of grinding feed by the method of damage accumulation
}

\author{
Timmo Gavrilov ${ }^{1 *}$ \\ ${ }^{1}$ Petrozavodsk State University, 185910 Petrozavodsk, Russia
}

\begin{abstract}
The efficiency of the process of grinding meat and bone feed depends on a number of design and technological parameters of the grinder operation. One of the most significant issues and at the same time the least studied one is the influence of the number of cycles of loading meat and bone feed with the working bodies of grinders on the particle size of the finished meat and bone feed. To study this issue, the authors proposed a method for modeling the process of grinding meat and bone feed using a stochastic B-model of cumulative damage, which is based on an understanding of the process under study at the macroscopic level and experimental data. The constructed B-model made it possible to determine the optimal number of cycles of loading meat and bone feed with the working bodies of the grinders, at which the required particle size of the finished meat and bone feed is $3 \ldots 5 \mathrm{~mm}$, and there is no unnecessary energy consumption of the grinding process. This number is equal to $12 \ldots 18$ cycles. The results obtained by the B-model are in good agreement with the experimental data. The constructed B-model of the grinding process of meat and bone feed will further help optimizing the operation of the cutting device and reducing energy consumption for the operation of the grinders as a whole.
\end{abstract}

\section{Introduction}

Grinding of materials is a very common and most energy-intensive process in many industries, and especially in agriculture. For example, it is a known fact that the grinding process all over the world consumes up to $50 \%$ of the electricity spent on the preparation of meat and bone feed for farm animals, and most of the electricity is consumed involuntarily and irrationally: it turns into heat and other types of energy $[1,2]$. At the same time, it is a known fact $[3,4]$ that nutrients are actively assimilated by farm animals only in crushed form, since the active surface of particles increases in crushed meat and bone feed. This helps to speed up the digestion process and the absorption of nutrients, which speaks about the importance of the grinding process in the technologies of preparing meat and bone feed for farm animals. In accordance with the zootechnical requirements, the particle size of the finished meat and bone feed must satisfy the maximum rate of the biological reaction, i.e. be within $3 \ldots .5 \mathrm{~mm}$. The efficiency of the grinding process primarily depends on the design and technological parameters of the grinder operation [5-7]. In this regard, researches

* Corresponding author: gavrilov@petrsu.ru 
aimed at studying the effect of the design and technological parameters of grinders on the particle size of the finished meat and bone feed, as well as the energy consumption of the process of grinding meat and bone feed are relevant.

In agriculture, various grinders are used for grinding meat and bone feed. A common feature of many of them is multistage grinding, which consists in multiple repetitions of cycles of loading meat and bone feed with the working bodies of the grinder (for example, the number of knife passes, the number of hammer blows).

The focus of most of the existing studies of the grinding process of meat and bone feed is aimed at studying the influence of geometric characteristics and speed modes of operation of the working bodies of grinders on the energy consumption of the grinding process [8-13]. At the same time, the question of the influence of the cycles of loading meat and bone feed with the working bodies of grinders on the particle size of the finished meat and bone feed, and, in turn, on the energy consumption of the process of grinding meat and bone feed, remains insufficiently studied, which is a significant problem that needs to be addressed.

In this regard, the purpose of this work is to study the effect of the number of loading cycles of meat and bone feed with the working bodies of grinders on the particle size of the finished meat and bone feed.

\section{Materials and methods}

The initial dimensions of meat and bone feed, its strength properties, stationarity of feeding fluctuate within significant limits. In connection with the random nature of the noted factors that form the evolution of the grinding process, it can be stated that the grinding process itself is random, and it is necessary to use probabilistic models to describe it. Such models can be stochastic models of cumulative damage [14-16], based on an understanding of the studied process at the macroscopic level and experimental data. The construction of a stochastic model of cumulative damage of the crushed material is based on the use of Markov chains embedded in the studied physical process. Cumulative damage is defined as an irreversible change in the particle size of meat and bone feed, leading to a gradual decrease in their size to the limiting state. In the initial period of the grinding process, not all meat and bone feeds have the same dimensions due to the technical capabilities and real conditions of their appearance. Therefore, the considered grinding processes of specific materials will exhibit different intensities of cumulative damage growth and, in this regard, there will be different times (number of hammer blows or cutting cycles) for reaching the limiting states that determine the end of the grinding process. In order to describe the probabilistic evolution of the increase in the number of particles of the crushed material (or a decrease in their size), it is necessary to determine the quantitative indicators of the behavior of the sampling function and use them to establish a probabilistic model of the cumulative damage of the crushed meat and bone feed.

Let's imagine that the process of damage accumulation occurs stepwise with jumps at the time of measurements. This is not entirely adequate to the process of formation of new particles of meat and bone feed, but it allows using a discrete model (discrete time and a finite number of states) to describe this phenomenon.

Let's break down the whole process of forming the sample function into discrete states $S_{1}, S_{2}, \ldots, S_{\mathrm{b}-1}, S_{\mathrm{b}}$ (Figure 1). 


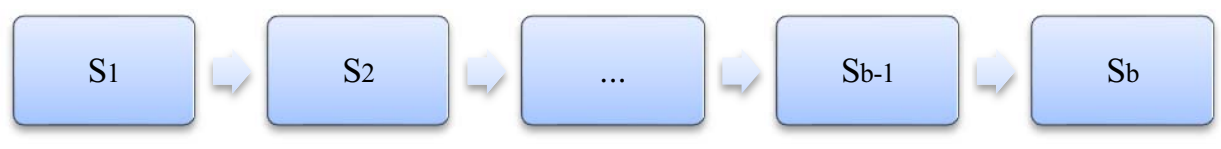

Fig. 1. Diagram of states of the process of cumulative damage of crushed meat and bone feed.

Let's assume that the initial value of the average particle size of meat and bone feed has a scatter relative to the average value at time $t=0$, which can be reflected by the mathematical expectation and standard deviation.

The damage is in the state $S_{1}$ until the moment when the impact force in accordance with the impact model [3] does not exceed the critical level. In practice, this abstraction means that all cyclic actions in the ongoing grinding processes (cutting, crushing), leading to a gradual accumulation of damage to feed particles, are reduced to impulse (instantaneous) periodic actions. If the value of the impact impulse exceeds the critical level, then the damage goes into state $S_{2}$, and this state is recorded during the next measurement of the average particle size. In cutting devices, the transition from the state $S_{\mathrm{i}}$ to the state $S_{2}$ is not necessary at each pass of the knife, since the probability of each subsequent particles of meat and bone feed getting under the influence of the knife is random and optional. Similarly, the damage remains in the state $S_{2}$ until the impact exceeds this critical level, and after exceeding it, it jumps into the state $S_{3}$, which is also recorded by direct measurement, and so on until it reaches the state $S_{\mathrm{b}}$ corresponding to the limiting state of the particle size of the crushed meat and bone feed (in accordance with the zootechnical requirements, the particle size of the finished meat and bone feed must satisfy the maximum rate of the biological reaction, i.e. be within $3 \ldots 5 \mathrm{~mm}$ ).

In the period between the states $S_{1}, S_{2}, \ldots, S_{\mathrm{b}}$, material damage accumulates as a result of the action of loading cycles on the material, and the intensity of the loading cycles is statistically similar and can be taken to be approximately constant. This means that what happens to the crushed meat and bone feed in one loading cycle is the same in the other loading cycle. In addition, it is meant that damage to meat and bone feed (change in particle size) is considered systematically at some intervals of time (or after a certain number of knife passes, the number of hammer blows), depending on the specific features of the grinding process, which will correspond to the conditional beginning and the end of the loading cycles.

The Markov property is manifested here as follows. The accumulation of damage in loading cycles depends only on the loading cycles and the state of damage at its beginning, and it is completely insignificant how the initial level of damage accumulation (particle size) is reached. Damage to the material, marked by the state $S_{\mathrm{j}}$ at the time of the beginning of the next loading cycles, can only increase and go to a state with a number one greater $S_{\mathrm{j}+1}$ and never return to the previous state.

Thus, it is assumed that there is a model in which all states are transitional, except for the last absorbing one (B-model). It should also be noted that the B-model does not say anything about quantitative changes among the processes occurring within each loading cycle, i.e. interactions are considered from the standpoint of physical laws. Such a model of cumulative damage to crushed meat and bone feed corresponds to a stationary Markov process with discrete time and state, embedded in a continuous physical process of accumulation of cumulative damage with single jumps. It is a matrix of transition probabilities, which, together with the vector of initial states that can always be determined, makes it possible to determine the probability of reaching the size level of the crushed meat 
and bone feeds specified by the states and to establish the function of the reliability of its achievement in the future.

\section{Results}

Based on the data of measurements of the average particle size of meat and bone feed after a certain loading cycle, presented in the studies [1, 17], a B-model was built with the parameters:

$$
r_{j}=\left\{\begin{array}{l}
1.99 \\
2.62 \\
3.46 \\
4.02
\end{array}\right\} ; j=\left\{\begin{array}{ll}
1,2 & b_{1}=3 \\
4,5 & b_{2}=6 \\
7,8,9 & b_{3}=10 \\
11,12,13,14 & b_{4}=15
\end{array}\right\}
$$

where $r_{\mathrm{j}}=P_{\mathrm{j}} / q_{\mathrm{j}}$;

$P_{\mathrm{j}}$ - the probability that the impact force (particle size) is below the critical level (control size) and the damage is in the same state;

$q_{\mathrm{j}}$ - the probability that the impact force (particle size) is higher than the critical level (control size), and the damage changes to a new state (implementation of a sequence of independent tests, where $q_{\mathrm{j}}$ - probability of success in one test);

$b_{1}, b_{2}, b_{3}$ - transient state of the process;

$b_{4}$ - absorbing state of the process corresponding to a failure in work, i.e. achieving the required result of the grinding process.

Table 1. The values of the experimental and design data of the model

\begin{tabular}{|c|c|c|c|c|}
\hline \multirow{2}{*}{$\begin{array}{c}\text { Average particle } \\
\text { size, mm }\end{array}$} & \multicolumn{2}{|c|}{ Average number of load cycles } & \multicolumn{2}{c|}{ Mean-square deviation } \\
\cline { 2 - 5 } & Experiment & B-model & Experiment & B-model \\
\hline 16.2 & 3.9 & 3.98 & 2.67 & 2.62 \\
\hline 10.5 & 8.1 & 8.64 & 4.32 & 4.45 \\
\hline 4.8 & 12.3 & 13.1 & 6.89 & 7.12 \\
\hline 1.7 & 24.0 & 25.6 & 13.48 & 13.51 \\
\hline
\end{tabular}

As a result of substitution of the values of the parameters of the B-model (1), depending on the determination of the average number of loading cycles and dispersion upon reaching certain specific values of the particle sizes of meat and bone feed during grinding, approximated data can be obtained, which, along with the experimental values, are presented in Table 1 and in Figure 2. 


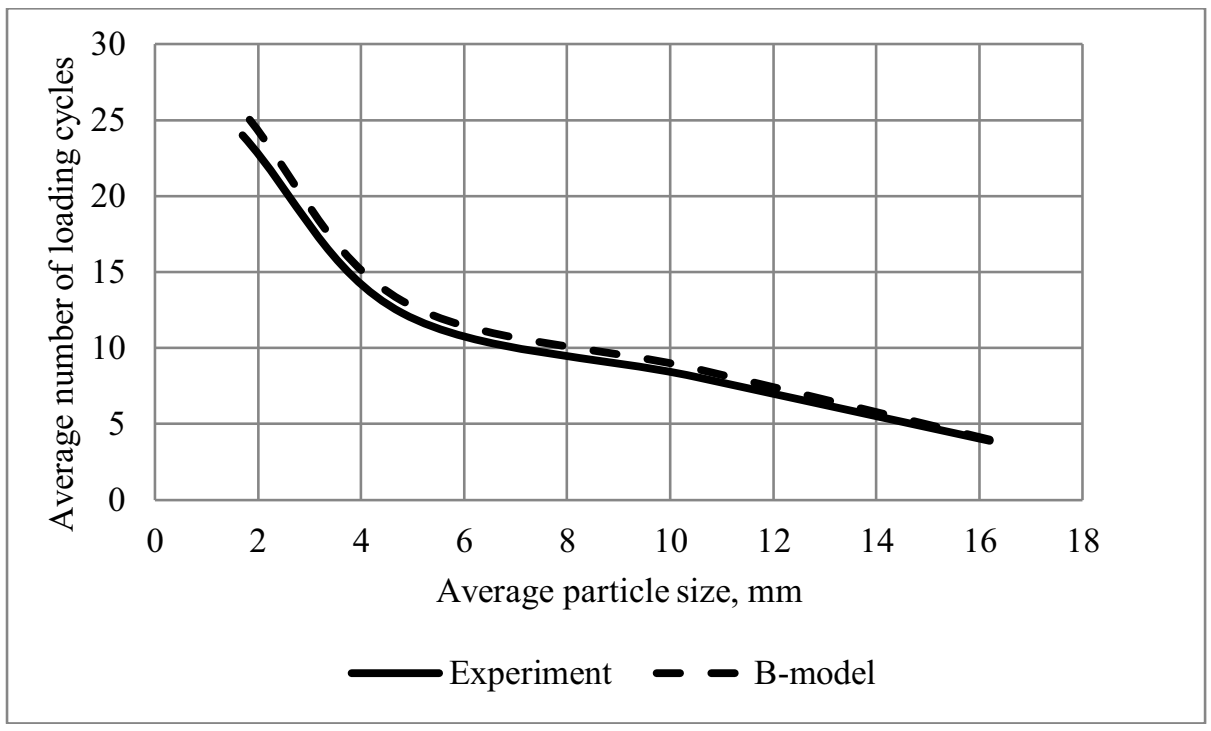

Fig. 2. The value of the experimental and design data of the B-model.

\section{Discussion}

Comparing the experimental data with the data of the B-model, one can note their satisfactory convergence. The matrix of transition probabilities $P_{\mathrm{x}}$, which, together with the row vector $P_{\mathrm{O}}$ of the initial probabilities of the particle size state, determines the probabilistic evolution of the process of changing the particle size of crushed meat and bone feed and, therefore, reaching the limiting size, has the form:

$$
\begin{aligned}
& P_{\mathrm{x}}=P_{\mathrm{O}} P^{\mathrm{x}}, x=0,1,2, \ldots \\
& P_{\mathrm{O}}=\left\{\pi_{1}, \pi_{2}, \ldots, \pi_{b-1,}, 0\right\} .
\end{aligned}
$$

As a first approximation in the matrix, we can take $\pi_{1}=1$, and then take into account the probabilities of the initial states of the row vector $P_{\mathrm{O}}$, i.e. deviations from the initial average size, then the possible states of $P_{\mathrm{O}}$ will be less than 1 , for example: $0.9 ; 0.8 ; \ldots$ to 0 .

The analysis of the obtained results (Figure 2) showed that the required particle size of the finished meat and bone feed (in accordance with zootechnical requirements) is achieved with an average number of cycles of loading meat and bone feed with the working bodies of the grinders equal to $12 \ldots 18$ cycles. Each excessive loading cycle leads to over-grinding of meat and bone feed and an increase in the energy consumption of the grinding process.

Thus, with the known B-model of the grinding process of meat and bone feed, the operation of the grinding unit can be further optimized, and the energy consumption for the operation of the grinder as a whole can be reduced. Structurally, changes in the grinding unit are possible, for example, a decrease in the number of knives.

\section{Conclusions}

In the course of studying the effect of the number of cycles of loading meat and bone feed with the working bodies of grinders on the particle size of the finished meat and bone feed: 
- the stochastic phenomenological B-model of cumulative damage to the grinding process of meat and bone feed was built;

- the size of the particles of the finished meat and bone feed was determined at different values of the cycles of their loading with the working bodies of the grinders;

- the optimal number of cycles of loading meat and bone feed with the working bodies of grinders was determined, at which the required particle size of the finished meat and bone feed is provided, and there is no unnecessary energy consumption of the grinding process. This number is equal to $12 \ldots 18$ cycles.

Continuation of research in this area through the construction and systematization of Bmodels of cumulative damage to the grinding process of various types of feed for farm animals will make it possible to develop recommendations for the design of cutting devices for grinders that reduce the energy consumption of the grinding process when the required particle size is reached.

\section{References}

1. T.A. Gavrilov, Resources and Technology, 14 (1), 9-17 (2017), https://doi.org/10.15393/j2.art.2017.3741

2. I.I. Ivanov, Yu.A. Plotnikov, Vestnik Chuvash SAA, 3, 84-89 (2020), https://doi.org/10.17022/j4jd-xz03

3. I. Ruhnke, I. Rohe, C. Kramer, F.G. Boroojeni, F. Knorr, A. Mader, E. Schulze, A. Hafeez, K. Neumann, R. Lowe, J. Zentek, Poultry Science, 94, 692-699 (2015), http://dx.doi.org/10.3382/ps/pev030

4. A.F.B. van der Poel, M.R. Abdollahi, H. Cheng, R. Colovic, L.A. den Hartog, D. Miladinovic, G. Page, K. Sijssens, J.F. Smillie, M. Thomas, W. Wang, P. Yu, W.H. Hendriks, Animal Feed Science and Technology, 270, 114692 (2020), https://doi.org/10.1016/j.anifeedsci.2020.114692

5. V. Bulgakov, S. Pascuzzi, S. Ivanovs, G. Kaletnik, V. Yanovich, Biosystems Engineering, 171, 155-164 (2018), https://doi.org/10.1016/j.biosystemseng.2018.04.021

6. F. Lyu, M. Thomas, W.H. Hendriks, A.F.B. van der Poel, Animal Feed Science and Technology, 261, 114347 (2020), https://doi.org/10.1016/j.anifeedsci.2019.114347

7. P. Adapa, L. Tabil, G. Schoenau, Biomass and Bioenergy, 35 (1), 549-561 (2011), https://doi.org/10.1016/j.biombioe.2010.10.004

8. M. Thomas, W.H. Hendriks, A.F.B van der Poel, Animal Feed Science and Technology, 240, 11-21 (2018), https://doi.org/10.1016/j.anifeedsci.2018.03.010

9. I. Cotabarren, M.P. Fernández, A.D. Battista, J. Piña, Powder Technology, 375, 433444 (2020), https://doi.org/10.1016/j.powtec.2020.08.016

10. A. Tekgüler, Emerging Materials Research, 10 (1), 128-135 (2021), https://doi.org/10.1680/jemmr.20.00275

11. J. Tong, S. Xu, D. Chen, M. Li, Journal of Bionic Engineering, 14 (1), 163-171 (2017), https://doi.org/10.1016/S1672-6529(16)60387-6

12. M.R. Abdollahi, V. Ravindran, B. Svihus, Animal Feed Science and Technology, 179, 1-23 (2013), https://doi.org/10.1016/j.anifeedsci.2012.10.011

13. J.S. Tumuluru, L.G. Tabil, Y. Song, K.L. Iroba, V. Meda, Biomass and Bioenergy, 60, 58-67 (2014), https://doi.org/10.1016/j.biombioe.2013.10.011 
14. H. Li, X. Luo, Y. Zhang, R. Xu, Engineering Fracture Mechanics, 245, 107566 (2021), https://doi.org/10.1016/j.engfracmech.2021.107566

15. W. Dong, S. Liu, S.J. Bae, Y. Cao, Reliability Engineering \& System Safety, 205, 107260 (2021), https://doi.org/10.1016/j.ress.2020.107260

16. Y. Feng, Q. Wang, D. Wu, W. Gao, F. Tin-Loi, Computer Methods in Applied Mechanics and Engineering, 372, 113371 (2020), https://doi.org/10.1016/j.cma.2020.113371

17. T.B. Stankevich, O.A. Anpilogova, G.I. Malinov, T.A. Gavrilov, Resources and Technology, 12 (2), 89-97 (2015), https://doi.org/10.15393/j2.art.2015.3101 